\title{
Soziale Marktwirtschaft - neu definiert
}

Florian Josef Hoffmann

Soziale Marktwirtschaft, was ist das? Haben wir überhaupt noch eine Soziale Marktwirtschaft? Das sind Fragen, die immer häufiger gestellt werden. Wir beklagen den Rückgang des Einflusses der Gewerkschaften, die systematische Ausbreitung der Leiharbeit, beobachten mit Sorge die Öffnung der EU-Grenzen nach Osten, sehen einen dramatischen Anstieg eines neuen Prekariats, das sich der $20 \%$-Marke nähert, zugleich wächst das Warenangebot in den Geschäften, genauer: das vielfältige Luxusangebot, für die Superreichen zu Wasser, zu Lande und in der Luft und das ein-fältige Discountangebot, die Grundausstattung für unsere Ärmeren (und preiswertes Ergänzungsangebot für unsere Wohlhabenderen, die es eigentlich nicht nötig haben).

Löblicherweise wird unsere Bundeskanzlerin Angela Merkel trotzdem nicht müde, ihre Reden mit der Forderung zu garnieren, dass die Soziale Marktwirtschaft als Exportartikel in die Welt hinausgetragen werden müsse, und: Seit dem Vertrag von Lissabon ist die Soziale Marktwirtschaft auch im EU-Vertrag verankert. Wir haben sie also, die Soziale Marktwirtschaft. Aber um sie in die Welt hinauszutragen, muss sie formuliert sein, muss das Rezept verständlich und vermittelbar sein, anders als die unbefriedigende Antwort auf der Homepage des Bundesministeriums für Wirtschaft und Technologie: „Die Idee geht zurück auf Ludwig Erhard. [...] Die zentrale Idee besteht darin, die Freiheit aller, die als Anbieter oder Nachfrager am Markt teilnehmen, zu schützen und gleichzeitig für sozialen Ausgleich zu sorgen." In Politikerreden ist dann von zwei Seiten einer Medaille die Rede, von einer marktwirtschaftlichen und einer sozialen, und der Wirtschaftswissenschaft hat sich der Begriff definitorisch überhaupt noch nicht erschlossen. Alle reden von Sozialer Marktwirtschaft und niemand weiß, was es ist!? In der Tat: Angela Merkels Wille, die Soziale Marktwirtschaft zu exportieren, scheitert an einer klaren Definition. Sie selbst reduziert ihre Botschaft auf die schlichte Kernwahrheit: „Der Sozialen Marktwirtschaft ist es gelungen, den Gegensatz von Kapital und Arbeit zu überwinden". Wie wahr! Aber wie ist das geschehen? Wie lautete das Rezept? Nachstehende Ausführungen liefern einen gedanklichen Ansatz:

\section{KAISERREICH UND WEIMAR}

Um sich einer Definition des Begriffs „Soziale Marktwirtschaft" zu nähern, ist ein kurzer Einblick in die Wirtschafts- und Ideengeschichte erforderlich: In den „Golden Twenties“ des 20. Jahrhunderts entwickelte sich derWohlfahrtsstaat schon prächtig. Die Entwicklung hatte ihren Grund: Sie war eine Fortsetzungsgeschichte der Vorkriegszeit. Deutschland hatte seit 1880, also schon mehr als 30 Jahre vor dem Ersten Weltkrieg, eine unvergleichliche Blüte erlebt. Die Bismarckschen Sozialgesetze, aber auch die flächendeckende Entstehung von Wirtschaftsverbänden, Gewerkschaften und Genossenschaften, etc. entwickelten sich im Gleichklang mit den Errungenschaften von Wissenschaft, Technik und Geisteswelt. Nach dem Ersten Weltkrieg erblühten sodann die Großgruppen des Kapitals und der Gewerkschaften erneut in korporatistischem Zusammenwirken mit dem Staat. Auch das Tarifvertragsgesetz von 1918 (!) tat seine Wirkung durch Stärkung der solidarischen Komponente. Abrupt unterbrochen wurden die Erfolge des neuen Wohlfahrtsstaates im Jahr 1929 durch ein importiertes Spekulationsdesaster (Wall-Street), auf das die staatliche Finanzpolitik dann fatalerweise die falsche Antwort gab (restriktive Finanzpolitik). Die unsäglichen Folgen sind bekannt.

Parallel dazu entstanden die kritischen Ideen der Freiburger Schule, später Ordoliberalismus genannt, gegründet von Walter Eucken und anderen. Der Politologe Philip Manow beschreibt das so: „Der
Ordoliberalismus entstand in der zweiten Hälfte der 1920er Jahre als Reaktion auf Inflation und Weltwirtschaftskrise. Der eigentliche Grund der Krise Weimars ist jedoch in den Augen dieser Autoren nicht wirtschaftlicher, sondern politischer Natur. Der Ordoliberalismus ist die wirtschaftspolitische Doktrin eines sich radikalisierenden, tief greifend ,statusverunsicherten " Bürgertums [...], das sich in der Weimarer Republik zunehmend wirtschaftlich aufgerieben sah zwischen den korporatistischen Großgruppen von Arbeit und Kapital, sich als politisch marginalisiert empfand, das sich aber schließlich auch konfessionell in die Enge getrieben fühlte. Träger der Doktrin ist eine bürgerlich-protestantische Bildungselite, die den Weimarer Korporatismus als handfeste ökonomische Bedrohung empfand und zugleich als Herausforderung eines konfessionellen Hegemonieanspruchs, den der lutherische Protestantismus seit 1871 für sich beansprucht hatte. Wichtiges Element der bürgerlichen Kritik am System von Weimar war der immer heftiger werdende Angriff auf den Weimarer Wohlfahrtsstaat. Auch diese Polemik hatte eine sozioökonomische, politische und konfessionelle Dimension. Denn von dem kräftig expandierenden Wohlfahrtsstaat schienen vor allem die christlichen (katholischen) und freien (sozialistischen) Gewerkschaften, entsprechend parteipolitisch das Zentrum und die Sozialdemokratie zu profitieren. Die Gewerkschaften waren gut in die Selbstverwaltung des Bismarckschen Sozialstaates integriert, während der soziale Protestantismus ein heterogenes, aber bei aller Heterogenität doch durchgängig (bildungs-)bürgerliches

Florian Josef Hoffmann, Rechtsanwalt, ist Leiter des European Trust Institute in Düsseldorf. Arbeitsschwerpunkte: Wettbewerbspolitik, Kartellrecht. e-mail: florian.hoffmann@eu-trust.org 
Projekt blieb. Ohne Mobilisierungserfolg in den Arbeiterschichten fehlte ihm die organisatorische Verbindung mit dem besonders auf die Interessenlage der Arbeitsschichten ausgerichteten deutschen Wohlfahrtsstaat.."

\section{MÜLLER-ARMACKS SOZIALE MARKTWIRTSCHAFT ALS GEMEINSAMES DACH}

Man kann sagen: Irgendwie gab es die Soziale Marktwirtschaft schon in Form des kräftig expandierenden Wohlfahrtsstaates, aber sie hatte noch keinen Namen. Das änderte der protestantische Münsteraner Ökonom Alfred Müller-Armack, der nicht nur den Begriff ersann, sondern auch noch das endgültige Konzept. Müller-Armack hatte die Bedeutung beider Komponenten erkannt, zugleich lag ihm für die Zeit nach dem Krieg daran, den konfessionellen Vorkriegsgegensatz aufzuheben. Er verband schon im Jahr 1942 gedanklich die widerstreitenden Ideenwelten des solidarischen Wirtschaftens (Historische Schule) und des Ordoliberalismus unter dem Begriff „Soziale Marktwirtschaft“ (in der Nazi-Zeit noch versteckt unter dem Begriff „textile Marktwirtschaft").

Manow schreibt über die Zeit nach 1950, als der Gegensatz naturgemäß erneut aufkam: „In den 1950er Jahren, als es erneut um die Frage der grundlegenden Ausrichtung des deutschen Wirtschaftsmodells ging, wiederholte sich die skizzierte Weimarer Konfliktkonstellation: Erhard oder Bismarck bzw. Erhard oder Adenauer ...? Ordoliberale Wirtschaftspolitik oder wohlfahrtsstaatlicher Sozialkatholizismus? Über die Nachkriegsordnung entzündete sich ein Konflikt innerhalb der CDU zwischen dem frankophilen und dem Bismarck'schen Wohlfahrtsstaat freundlich gesonnenen katholischen Flügel der CDU und einem protestantischen, atlantischen und dabei hochgradig sozialstaatskritischem Flügel, wie er von Erhard repräsentiert wurde. “2 Das war in der damaligen Realität nicht schädlich, weil sich das „Wirtschaftswunder“ allerprächtigst entwickelte. Aber was nicht nur hintergründig schwelte, war eine Art kleiner Religionskrieg, also eine - bis heute unaufgelöste - denkbar unerfreuliche Situation.

Wie nun lässt sich der Gegensatz der Systeme vereinen, der Wille Müller-Armacks verwirklichen, der überwältigende Erfolg der Sozialen Marktwirtschaft erklä- ren, ohne die beiden Ansätze als Gegensätze zu begreifen?

\section{SOZIALE MARKTWIRTSCHAFT IN NEUER DEFINITION}

Der Zugang zu einer klaren, widerspruchsfreien Definition erfolgt über die Zuordnung der vermeintlich gegensätzlichen Ideenwelten des solidarischen Wirtschaftens zum Prinzip der Solidarität einerseits und des Ordoliberalismus zum Prinzip der Subsidiarität andererseits, nicht vergessend, dass beides schon Kernprinzipien der Sozial-Enzyklika „rerum novarum“ (1891) des Papstes Leo XIII waren. Die beiden Systeme bzw. Prinzipien hatten sich in der gemeinsamen realen Umsetzung schon zu dessen Lebzeiten in Deutschland zusammengefunden (wo sie von seinem Ideengeber, dem Münsteraner und späteren Bischof von Mainz, Wilhelm Emmanuel von Ketteler, beobachtet und analysiert wurden) und sich sodann trotz zweier Weltkriege und Katastrophen über die „Golden Twenties“ und das „Wirtschaftswunder" fortentwickelt:

Das solidarische Wirtschaften lieferte unter dem Prinzip der Solidarität den Wohlstand. Das Prinzip bedeutet konsensorientiertes Wirtschaften (Wettbewerb, nicht Wettkampf!) in freiheitlicher Nutzung aller Arten vertraglicher Bindungen. Es dient der Schaffung maximaler Prosperität bei optimaler Verteilung (Tarifverträge für Dienste aller Art). Solidarische Regelungen sind nicht nur bei den Gewerkschaften förderlich, sondern auch bei den Betrieben und Unternehmen (Verbände und Genossenschaften), weil sie helfen, die Wertschöpfung zu maximieren und zugleich auch schwächeren Marktteilnehmern eine Existenzchance bieten (Verbandssatzung, Handwerksordnung, Preis-, Mengen-, Qualitäts- und Regionalregulierung). Die dynamische Mehrung des Wohlstands erfolgt durch positive Motivation aller Anbieter, auch der Arbeiter (Anbieter von Arbeit) und in der Summe dann zusätzlich dadurch, das möglichst viele Leistungswillige im Wettbewerb eingebunden und produktiv tätig sind - was auch bedeutet, dass die Solidargemeinschaften offen sein müssen. Viele Fehler früherer Gemeinschaften, wie z. B. die zu streng abgeschotteten Zünfte, dürfen nicht wiederholt werden.

Der Ordoliberalismus liefert sodann obrigkeitlich aber zurückhaltend, eben subsidiär, dort den sozialen Ausgleich, wo der privatrechtliche Ausgleich nicht greift, nämlich außerhalb der Solidarsysteme, beispielsweise bei Alten, Schwachen und Bedürftigen. Er sorgt außerdem dafür, dass der Staat die gesetzlichen Regeln sowohl für die privaten als auch die öffentlichrechtlichen Solidarsysteme schafft, aber eben in beiden Fällen unter Beachtung des Subsidiaritätsprinzips.

Beides zusammen bedeutet: Im System der Sozialen Marktwirtschaft ist sowohl der Idee des wohlfahrtsstaatlichen Sozialkatholizismus wie der des Ordoliberalismus konkret Rechnung getragen! Die nachstehende systematische Zuordnung lässt die eindeutige und kollisionsfreie Kombination der beiden Ansätze erkennen.

\section{MERKMALE DES SOLIDARISCHEN WIRTSCHAFTENS:}

(1) Sein Kernmerkmal ist die Solidarität, die Verbundenheit untereinander bzw. die Unterstützung anderer hinsichtlich ihrer Ziele, Aktivitäten und Ideen.

(2) Solidarität wirkt horizontal von Subjekt zu Subjekt, von Mensch zu Mensch, in jeweils der gleichen Ebene.

(3) Solidarisches Wirtschaften wird privatrechtlich organisiert. Es geschieht fern jeder staatlichen Aktivität, bewegt sich aber in Rechtsräumen, die der Rechtsstaat für private Initiativen eröffnet. Der Einzelne ordnet sich darin der freiwilligen Vereinbarung, den allgemeinen Gesetzen und der Zivilgerichtsbarkeit unter.

(4) Solidarisches Wirtschaften ist eigeninitiativ und freiwillig. Es entwickelt und organisiert sich in privatrechtlichen Organisationen, d.h. in Form von Verbänden, Vereinen, Gewerkschaften, Genossenschaften, Kartellen, etc.

(5) Grundgesetzlich geschützt ist die Privatautonomie in Form liberaler Gestaltungsrechte (Koalitionsfreiheit, Vereinigungsfreiheit, Vertragsfreiheit).

1 Manow, Ph. (2010): Die soziale Marktwirtschaft als interkonfessioneller Kompromiss, in: Ethik und Gesellschaft (1), S. 4, http://www.ethik-und-gesellschaft.de/mm/EuG-1-2010_Manow.pdf. 


\section{KOMPLEMENTÄRE PRÄMISSEN \\ EINER ORDOLIBERALEN \\ WIRTSCHAFTSPOLITIK:}

(1) Kernmerkmal ordoliberaler Wirtschaftspolitik ist die Subsidiarität, die eigenverantwortliches Handeln vor das Handeln des Staates stellt.

(2) Das Prinzip der Subsidiarität wird dabei vertikal realisiert, also im Verhältnis Staat zu Bürger von oben nach unten, also von Ebene (Regierung/Verwaltung) zu Ebene (Bürger).

(3) Geregelt wird das Prinzip öffentlichrechtlich. Das gilt sowohl für Eingriffe als auch für staatliche Zuwendungen, aber auch für staatliche Solidarsysteme (Zwangs-Solidarität: Arbeitslosen-, Unfall-, Rentenversicherung). Gerichtliche Kontrollinstanzen sind Verwaltungsgerichte.

(4) Verwirklicht bzw. angewandt wird das Prinzip bürokratisch, also hoheitlich zur Regelung von Einzelschicksalen im Rahmen der Sozialhilfen (auch: Bafög, Wohngeld, etc.) und der staatlichen Solidarsysteme.

(5) Verfassungsrechtlich ist das Subsidiaritätsprinzip ein Garant des liberalen Rechtsstaats gegen zu viel staatliche Regulierung und zugleich Grenze des öffentlichrechtlichen Sozialstaats.

Die Merkmale ließen sich tabellarisch gegenüberstellen. Jedenfalls: Der aus beiden „Welten“ bzw. Prinzipien kombinierte neue Wohlfahrtsstaat, von Müller-Armack unter das Dach „Soziale Marktwirtschaft" gestellt, ist eine geniale Konstruktion, die sowohl dem sozialen, wie auch dem liberalen Aspekt in hohem Maße Rechnung trägt. In der realen Umsetzung gibt es ohnehin keinen „Religionskrieg“ - in der theoretischen Auseinandersetzung löst er sich jetzt in Wohlgefallen auf. Weshalb diese Erkenntnis nicht früher gewonnen wurde, erklärt sich aus der Entstehungsgeschichte:

\section{URSPRUNG UND HEUTIGE KONFLIKTE}

Wenngleich zufällig, so war das Zustandekommen im Grunde doch der dynamischen Zeit des späteren 19. Jahrhunderts und einem gemeinsamen Kampf von Papst Leo XIII und Bismarck gegen den Sozialismus/Marxismus geschuldet. Papst Leo XIII formulierte mit der erwähnten Enzyklika, in Verteidigung der christlichen Werteordnung gegen die materialistischkommunistische Weltanschauung, den „dritten Weg“. Bismarck kämpfte auf preussisch-obrigkeitliche Weise per Gesetz gegen die Sozialisten durch Einführung von Systemen der sozialen Sicherung. So institutionalisierte er die staatlichen Sozialsysteme als solidar-subsidiäre Komponenten einer sozialen Marktwirtschaft, mit der er eigentlich die Sozialisten zu bekämpfen gedachte. Zugleich wurden im Kaiserreich die Solidarsysteme der Unternehmer, Kartelle und Verbände befürwortet und gefördert. Darüber hinaus hatte man auch die Segnungen eines $\mathrm{Zu}$ sammenwirkens von Staat und Wirtschaft erkannt (Korporatismus). Alles zusammen beförderte einen bisher unbekannten, ungeahnten wirtschaftlichen Wohlstand.

Die Historische Schule, also die wissenschaftliche Disziplin, die die Entwicklung in Deutschland im 19. Jahrhundert geistig begleitete, versank zwischen den Weltkriegen in der Bedeutungslosigkeit. Der Ordoliberalismus erstarkte in den 1930er Jahren, auf ihn konnte und wollte sich Müller-Armack nicht alleine stützen. Die innere Widersprüchlichkeit der beiden Ideenwelten vermochte er jedoch nicht aufzulösen. Infolgedessen einigten sich die Protagonisten der Sozialen Marktwirtschaft in der Nachkriegszeit darauf, zusammenzuhalten, nach außen Einigkeit zu zeigen, jedoch unbestimmt zu bleiben, was den Inhalt des Begriffs "Soziale Marktwirtschaft" anlangte. Der Konflikt, dessen Vermeidung sich MüllerArmack auf die Fahnen geschrieben hatte, ist deshalb bis heute unaufgelöst und unterschwellig präsent - letztlich der Grund, weshalb niemand so recht weiß oder beschreiben kann, was Soziale Marktwirtschaft wirklich ist.
Die geistige Unschärfe blieb nicht ohne Folgen und Schäden. Denn objektiv betrachtet betreibt der Ordoliberalismus in Deutschland noch heute einseitig im Sinne Bismarcks und Erhards die Verdrängung der Solidarsysteme der Arbeiterschaft, der Gewerkschaften. Und auch die Beseitigung der Solidarsysteme der Unternehmen Kartelle bzw. Verbände - mittels Kartellrecht setzt sich in beängstigender Weise durch, weil sich der Ordoliberalismus über den Begriff der „Wettbewerbsordnung“ die maßgebliche wirtschaftspolitische Kompetenz erobert hat. Die ordoliberalen Gedanken - obwohl in der Wissenschaft von den Neoklassikern verdrängt - sind überstark präsent, weil Ludwig Erhard als „Vater des Wirtschaftswunders“ zu Unrecht Haupt-Nutznießer im Ansehen wurde (Müller-Armack war „nur“ sein Staatssekretär).

Das Kartellrecht, ein von Erhard aus dem anglo-amerikanischen Rechtsraum importiertes Rechtsgebilde, das solidarische Abreden generell verbietet und das schon in seiner Überschrift das genaue Gegenteil einer Wettbewerbsordnung beschreibt („Gesetz gegen Wettbewerbsbeschränkungen“, also für schrankenlosen Wettbewerb), steht im absoluten Gegensatz zu einem der wesentlichen Prinzipien der Sozialen Marktwirtschaft. Erst wenn dieser Grundirrtum politisch berichtigt ist, könnte das Gleichgewicht von solidarischer Wirtschaft und Ordoliberalismus, von Solidarität und Subsidiarität wiederhergestellt werden, erst dann hätte die Soziale Marktwirtschaft eine Chance, das zu werden, was sie schon lange verdient hat, nämlich: Exportartikel, erst dann bekäme sie die Chance, sich und dem Wirken Müller-Armacks die lange verdiente Weltgeltung zu verschaffen.

Der weitere Weg zur Durchsetzung dieses Zieles zeichnet sich auch schon ab, weil, wie erwähnt, seit dem Vertrag von Lissabon die Soziale Marktwirtschaft im EU-Vertrag wörtlich verankert ist, wo es in Titel I (Gemeinsame Bestimmungen), Art. 3 Abs. 3 EUV, heißt, dass die Union hinwirkt auf „[...] eine in hohem Maße wettbewerbsfähige soziale Marktwirtschaft, die auf Vollbeschäftigung und sozialen Fortschritt abzielt [...]“. 